\title{
Modeling of unsteady flow around well with hydraulic fracture
}

\author{
A.A. Khisamov, I.L. Khabibullin \\ Bashkir State University, Department of Applied Physics, Ufa, Russia
}

\begin{abstract}
At present, hydraulic fracturing technologies are widely used for intensification of oil and gas recovery from reservoirs with hard-torecover reserves. Modeling the processes of flow through porous reservoirs with hydraulic fractures has beenfairly completely developed in the steadystate flow approximation. Non-stationary processes of pressure distribution are considered as applied to the theory of hydrodynamic well survey methods, in which asymptotically limited intervals of coordinate and time variations are taken into account, i.e., distances of the order of the well radius and time significantly shorter than the characteristic time of the flow through the porous medium. At the same time, in reservoirs with hard-torecover reserves (low-permeability reservoirs and high-viscosity oils), the duration of unsteady processes of pressure redistribution can be of the same order as the characteristic time of flow through the reservoir. This study presents new analytical solutions to the problem of the redistribution of non-stationary pressure in the vicinity of a well penetrated by a vertical fracture. The scientific novelty of the study lies in the fact that the model used takes into account, first, the fluid compressibility in the fracture and, secondly, the fluid flow not only through the fracture, but also through the porous reservoir. The solutions to the problems are constructed using the Laplace transform technique. In particular cases, the expressions known in literature follow from the solutions obtained. The analytical solutions obtained are analyzed, which makes it possible to determine the main characteristic features of the flow processes of around a porous medium.
\end{abstract}

\section{Formulation of the problem}

In a reservoir saturated with low-compressible homogeneous fluid, there is a well intersected by a symmetrical vertical hydraulic fracture throughout the entire thickness of the reservoir. The hydraulic connection between the formation and the well is implemented only through the lateral surface of the fracture, since the width (opening) of the fracture is much smaller than its length. It is assumed that initially the pressure in the reservoir and the fracture is the same, and at $t=0$ the well is put into production. Then, an unsteady filtration flow is created around the well in the fracture and in the formation. Taking into account the symmetry of the geometry of the problem with respect to the well and the fracture, only one fourth of the filtration area is considered (Fig. 1).

Since the fracture width is much less than the length, the flow in the fracture is assumed to be one-dimensional, directed along the x-axis. The flow in the formation is directed along the $y$-axis perpendicular to the fracture lateral surface as shown in Fig. 1. The scheme 
corresponds to the so-called bilinear flow regime, which is a combination of two linear mutually perpendicular flows simultaneously existing in the fracture and in the reservoir [1, $2,3,4,5]$.

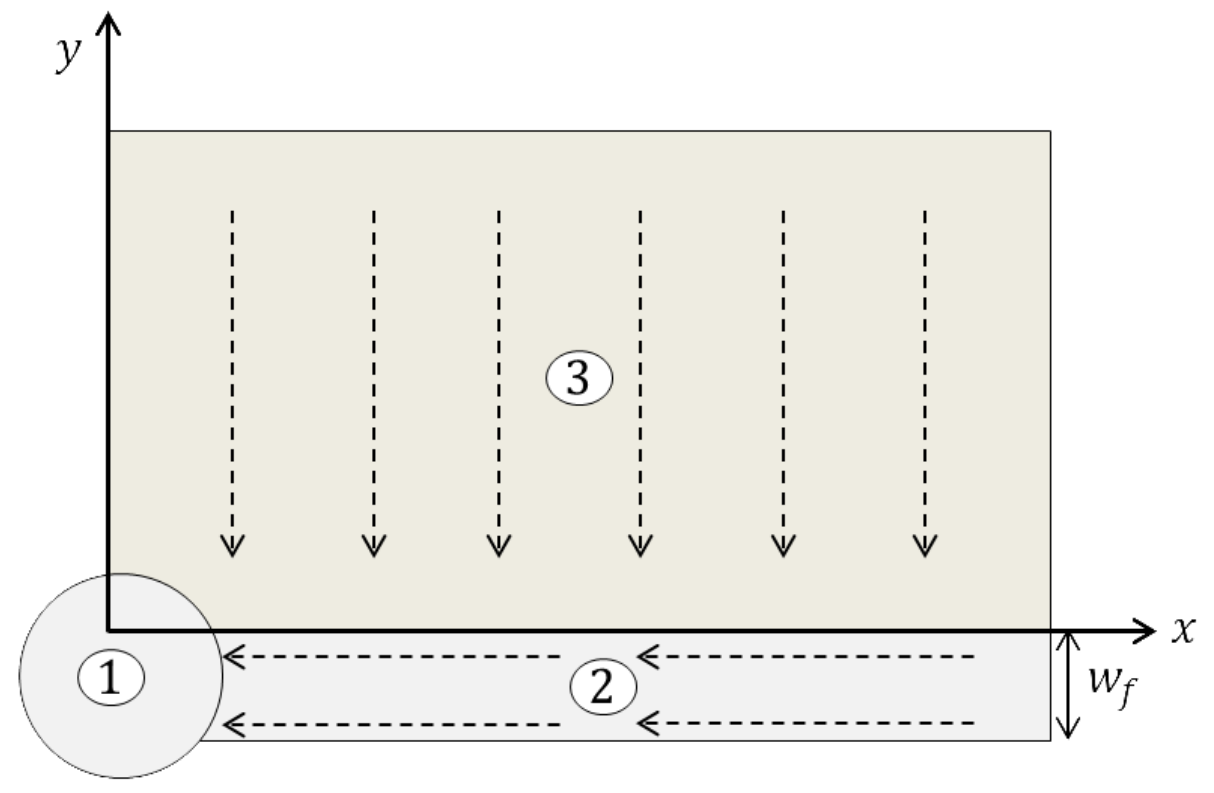

Fig.1. Diagram of the flow region: the well (1), the fracture (2), and the reservoir (3).

The pressure distributions over the reservoir and the fracture can be described by the equations:

$$
\begin{aligned}
& \frac{\partial P_{r}}{\partial t}=\kappa_{r} \frac{\partial^{2} P_{r}}{\partial y^{2}} \quad 0 \leq x \leq \infty, \quad 0 \leq y \leq \infty \\
& \frac{\partial P_{f}}{\partial t}=\kappa_{f} \frac{\partial^{2} P_{f}}{\partial x^{2}}+\left.\frac{\kappa_{f}}{w_{f}} \frac{k_{r}}{k_{f}} \frac{\partial P_{r}}{\partial y}\right|_{y=0} \quad 0 \leq x \leq \infty, \quad-w_{f} \leq y \leq 0 .
\end{aligned}
$$

Where $P$ is the pressure, $x$ and $y$ are the coordinates, $t$ is time, the subscripts $r$ and $f$ relate to the reservoir and the fracture, respectively, $\mathrm{K}$ is the piezoconductivity coefficient, $k$ is the permeability, and $w_{f}$ is the fracture half-width.

The initial pressure distribution is

$$
P_{r}(x, y, t=0)=P_{f}(x, t=0)=P_{0} .
$$

On the fracture surface (reservoir-fracture interface), the condition of equality of the pressures must be met:

$$
P_{r}(x, y=0, t)=P_{f}(x, y=0, t) .
$$

On the impermeable boundaries, the conditions are as follows:

$$
\frac{\partial P_{r}(x, y=\infty, t)}{\partial y}=0
$$




$$
\frac{\partial P_{f}(x=\infty, y, t)}{\partial x}=0, \quad \frac{\partial P_{f}\left(x, y=-w_{f}, 0\right)}{\partial y}=0
$$

On the surface of the intersection of the fracture with the well, the condition is met, stating that the well is operating at a given pressure $P_{c}$ or a given flow rate $Q$ :

$$
\begin{aligned}
& P_{f}(x=0, t)=P_{c} . \\
& \frac{k_{f} h_{r} w_{f}}{\mu} \frac{\partial P_{f}(x=0, t)}{\partial x}=Q .
\end{aligned}
$$

Where $\mu$ is the fluid viscosity, $Q$ is the part of the flow rateentering the well from the considered porous flow area, $x_{f}$ is the fracture half-length, and $h_{r}$ is the reservoir thickness.

Accordingly, we have the first ((1)-(6)) or the second ((1)-(5), (7)) boundary-value problems. In dimensionless variables

$$
\bar{P}_{r}=\frac{P_{r}-P_{0}}{P^{*}}, \quad \bar{P}_{f}=\frac{P_{f}-P_{0}}{P^{*}}, \quad P_{1}^{*}=P_{c}-P_{0}, \quad P_{2}^{*}=\frac{Q \mu}{k_{r} h_{r}}, \quad \bar{y}=\frac{y}{x_{f}}, \quad \bar{x}=\frac{x}{x_{f}}, \quad \bar{t}=t \frac{\kappa_{r}}{x_{f}^{2}}
$$

these problems take the form:

$$
\begin{gathered}
\frac{\partial \bar{P}_{r}}{\partial \bar{t}}=\frac{\partial^{2} \bar{P}_{r}}{\partial \bar{y}^{2}}, \\
\frac{\partial \bar{P}_{f}}{\partial \bar{t}}=a \frac{\partial^{2} \bar{P}_{f}}{\partial \bar{x}^{2}}+\left.b \frac{\partial \bar{P}_{r}}{\partial \bar{y}}\right|_{\bar{y}=0,}, \\
\overline{P_{r}}(\bar{x}, \bar{y}, \bar{t}=0)=\bar{P}_{f}(\bar{x}, \bar{t}=0)=0, \\
\bar{P}_{r}(\bar{x}, \bar{y}=0, \bar{t})=\bar{P}_{f}(\bar{x}, \bar{t}), \\
\bar{P}_{f}(\bar{x}=0, \bar{t})=1, \\
\frac{\partial \bar{P}_{f}(\bar{x}=0, \bar{t})}{\partial \bar{x}}=\frac{b}{a}, \\
\bar{P}_{i}(\bar{x} \rightarrow \infty, \bar{y} \rightarrow \infty, \bar{t})=0 \quad(i=r, f) .
\end{gathered}
$$

where $a=\mathrm{bl}_{f} / \mathrm{bl}_{r}, \quad b=a\left(k_{r} / k_{f}\right)\left(x_{f} / w_{f}\right)$, and the value of $a / b$ coincides with the dimensionless fracture conductivity, first introduced in and widely used in literature. The quantities $P_{1}^{*}$ and $P_{2}^{*}$ refer to the first and second boundary value problems, respectively.

The above model is invariant with respect to changes in the signs of the well flow rate and drawdown. Therefore, it makes is possible to study both the process of withdrawing 
fluid from the reservoir through the fracture and the well, and the process of pumping fluid into the reservoir through a well with a hydraulic fracture.

The solution to the first boundary value problem was considered in detail in [6], therefore, here we present only the final form of the solution. In this work, two types of solutions were obtained. The first solution is:

$$
\begin{gathered}
\bar{P}_{f}(\bar{x}, \bar{t})=1-\frac{1}{\pi} \int_{0}^{\infty} \exp \left(-\rho \bar{t}-f_{1}(\rho) \bar{x}\right) \sin \left[f_{2}(\rho) \bar{x}\right] \frac{d \rho}{\rho} \\
\bar{P}_{r}(\bar{x}, \bar{y}, \bar{t})=1-\frac{1}{\pi} \int_{0}^{\infty} \exp \left(-\rho \bar{t}-f_{1}(\rho) \bar{x}\right) \sin \left[f_{2}(\rho) \bar{x}+\rho \sqrt{\bar{y}}\right] \frac{d \rho}{\rho} \\
f_{1}(\rho)=\left[\frac{\sqrt{\rho^{2}+b^{2} \rho}-\rho}{2 a}\right]^{1 / 2}, \quad f_{2}(\rho)=\left[\frac{\sqrt{\rho^{2}+b^{2} \rho}+\rho}{2 a}\right]^{1 / 2} .
\end{gathered}
$$

Another form of the solution can be expressed by the formulas

$$
\begin{gathered}
\bar{P}_{f}(\bar{x}, \bar{t})=\frac{\bar{x}}{\sqrt{\pi a \bar{t}}} \int_{0}^{1} \exp \left(-\frac{\bar{x}^{2}}{4 a \bar{t} \rho^{2}}\right) \operatorname{erfc}\left(\frac{\rho^{2} b \sqrt{\bar{t}}}{2 \sqrt{1-\rho^{2}}}\right) \frac{d \rho}{\rho^{2}} . \\
\bar{P}_{r}(\bar{x}, \bar{y}, \bar{t})=\frac{\bar{x}}{\sqrt{\pi a \bar{t}}} \int_{0}^{1} \exp \left(-\frac{\bar{x}^{2}}{4 a \bar{t} \rho^{2}}\right) \operatorname{erfc}\left(\frac{\bar{y}+\rho^{2} b \bar{t}}{2 \sqrt{\bar{t}\left(1-\rho^{2}\right)}}\right) \frac{d \rho}{\rho^{2}} .
\end{gathered}
$$

Where erfc $(\xi)$ is the complementary error function

$$
\operatorname{erfc}(\xi)=\frac{2}{\sqrt{\pi}} \int_{\xi}^{\infty} \exp \left(-u^{2}\right) d u
$$

Expressions (17), (18) must be identical to (15), (16), since they represent solutions to the same problem, constructed by the Laplace transform method, but their transition from images to the originals is implemented by different methods. When deriving expressions (15) and (16), we used Mellin's theorem, and (17) and (18) were obtained using the general rules of the Laplace transform.

\section{Solution to the problem}

We will construct the solution of the second boundary value problem by the Laplace transform of the variable $t(L$ is the symbol of the Laplace transform)

$$
L[\bar{P}(\bar{x}, \bar{y}, \bar{t})]=\overline{\bar{P}}(\bar{x}, \bar{y}, \mathrm{~s})=\int_{0}^{\infty} \bar{P}(\bar{x}, \bar{y}, \bar{t}) \exp (-s \bar{t}) d \bar{t}
$$

In the Laplace transform images, the problem (8)-(14) takes the form: 


$$
\begin{gathered}
\frac{d^{2} \overline{\bar{P}}_{r}}{d y^{2}}=s \overline{\bar{P}}_{r}, \\
\frac{d^{2} \overline{\bar{P}}_{f}}{d x^{2}}-\frac{s}{a} \overline{\bar{P}}_{f}+\left.\frac{b}{a} \frac{d \overline{\bar{P}}_{r}}{d y}\right|_{y=0}=0 \\
\overline{\bar{P}}_{r}(x, y=0, s)=\overline{\bar{P}}_{f}(x, s), \\
\frac{d \overline{\bar{P}}_{f}(0, s)}{d x}=\frac{b}{a} \frac{1}{s}, \\
\overline{\bar{P}}_{i}(x \rightarrow \infty, y \rightarrow \infty)=0 \quad(i=r, f) .
\end{gathered}
$$

Thus, in the Laplace image space, we have a system of ordinary differential equations with respect to $\overline{\bar{P}}_{f}$ and $\overline{\bar{P}}_{r}$.

The solution to this system is found by standard methods and is presented in the following form:

$$
\begin{aligned}
& \overline{\bar{P}}_{f}(\bar{x}, s)=-\frac{b}{a} \frac{\exp (-\bar{x} \cdot \delta)}{s \delta}, \\
& \overline{\bar{P}}(\bar{x}, \bar{y}, s)=-\frac{b}{a} \frac{\exp (-\bar{x} \cdot \delta-\bar{y} \sqrt{s})}{s \delta}, \\
& \delta \equiv\left[\frac{s}{a}+\frac{b}{a} \sqrt{s}\right]^{1 / 2}
\end{aligned}
$$

The final solutions of the problems are obtained by converting expressions (24), (25) to the originals.

For the convenience of finding the originals, expression (24) is presented in the form:

$$
\overline{\bar{P}}_{f}(\bar{x}, s)=-\frac{1}{\sqrt{a} b \sqrt{s}} L[V(\bar{x}, \bar{t})], \quad L[V(\bar{x}, \bar{t})]=\frac{b}{\sqrt{s} a} \frac{\exp (-\bar{x} \delta)}{\delta}
$$

Using the convolution theorem and the Laplace transform inversion formula [12] ( $L^{-1}$ is the symbol of the inverse Laplace transform)

$$
L^{-1}\left(\frac{1}{\sqrt{s}}\right)=\frac{1}{\sqrt{\pi t}},
$$

from (26) we have

$$
\bar{P}_{f}(\bar{x}, \bar{t})=-\frac{1}{\sqrt{a} b} \int_{0}^{\bar{t}} \frac{1}{\sqrt{\pi(\bar{t}-\tau)}} V(\bar{x}, \tau) d \tau .
$$

Thus, it is necessary to find $V(\bar{x}, \bar{t})$. 
Applying the similarity theorem [7] to the second of the expressions (26), we find

$$
\left.L V\left(\bar{x}, b^{2} \bar{t}\right)\right]=\frac{1}{\sqrt{s}} \frac{\exp \left[-\frac{b \bar{x}}{\sqrt{a}}(s+\sqrt{s})^{1 / 2}\right]}{\sqrt{s+\sqrt{s}}} .
$$

For finding $V(\bar{x}, \bar{t})$ in (27), we will use the following rule of operational calculus:

$$
L^{-1}\left[\frac{g(s+\sqrt{s})}{\sqrt{s}}\right]=\frac{1}{\sqrt{\pi}} \int_{0}^{\bar{t}} \frac{1}{\sqrt{\bar{t}-u}} \exp \left(-\frac{u^{2}}{4(\bar{t}-u)}\right) f(u) d u, \quad f(u)=L^{-1}[g(s)]
$$

Then,

$$
V\left(\bar{x}, b^{2} \bar{t}\right)=\frac{1}{\pi} \int_{0}^{b^{2} \bar{t}} \exp \left(-\frac{u^{2}}{4\left(b^{2} \tau-u\right)}-\frac{b^{2} x^{2}}{4 a u}\right) \frac{d u}{\sqrt{b^{2} \tau-u} \sqrt{u}} .
$$

Taking this expression into account, we can represent (27) in the form

$$
\bar{P}_{f}(\bar{x}, \bar{t})=-\frac{1}{\sqrt{a} b \pi \sqrt{\pi}} \int_{0}^{\bar{t}} \frac{1}{\sqrt{\bar{t}-\tau}} \int_{0}^{b^{2} \tau} \exp \left(-\frac{u^{2}}{4\left(b^{2} \tau-u\right)}-\frac{b^{2} \bar{x}^{2}}{4 a u}\right) \frac{d u d \tau}{\sqrt{b^{2} \tau-u} \sqrt{u}} .
$$

Changing here the order of integration and evaluating the inner integral

$$
\int_{u / b^{2}}^{\bar{t}} \frac{1}{\sqrt{\left(b^{2} \tau-u\right)(\bar{t}-\tau)}} \exp \left(-\frac{u^{2}}{4\left(b^{2} \tau-u\right)}\right) d \tau=\frac{\pi}{b} \operatorname{erfc}\left(\frac{u}{2 \sqrt{b^{2} \bar{t}-u}}\right) .
$$

we obtain an expression for the pressure distribution in the fracture

$$
\bar{P}_{f}(\bar{x}, \bar{t})=-\frac{1}{\sqrt{a} \sqrt{\pi}} \int_{0}^{b^{2} \bar{t}} \exp \left(-\frac{b^{2} \bar{x}^{2}}{4 a u}\right) \operatorname{erfc}\left(\frac{u}{2 \sqrt{b^{2} \bar{t}-u}}\right) \frac{d u}{\sqrt{u}}
$$

In (28), for the convenience of numerical calculations, we replace the integration variable $u$ by $\rho^{2} b^{2} \bar{t}$. Then we have

$$
\bar{P}_{f}(\bar{x}, \bar{t})=-\frac{2 b \sqrt{\bar{t}}}{\sqrt{a} \sqrt{\pi}} \int_{0}^{1} \exp \left(-\frac{\bar{x}^{2}}{4 a \bar{t} \rho^{2}}\right) \operatorname{erfc}\left(\frac{\rho^{2} b \sqrt{t}}{2 \sqrt{\left(1-\rho^{2}\right)}}\right) d \rho .
$$

Performing similar operations, from (25), we obtain an expression for the pressure distribution in the reservoir:

$$
\bar{P}_{r}(\bar{x}, \bar{y}, \bar{t},)=-\frac{2 b \sqrt{t}}{\sqrt{a} \sqrt{\pi}} \int_{0}^{1} \exp \left(-\frac{\bar{x}^{2}}{4 a \bar{t} \rho^{2}}\right) \operatorname{erfc}\left(\frac{\rho^{2} b \bar{t}+\bar{y}}{2 \sqrt{\bar{t}\left(1-\rho^{2}\right)}}\right) d \rho .
$$




\section{Analysis of the solution}

Setting $\bar{x}=0$ in (32), we can find the pressure at the bottom of the well:

$$
\bar{P}_{f}(\bar{x}=0, \bar{t})=-\frac{1}{\sqrt{\pi a}} \int_{0}^{b^{2} \bar{t}} \operatorname{erfc}\left(\frac{u}{2 \sqrt{b^{2} \bar{t}-u}}\right) \frac{d u}{\sqrt{u}}
$$

The asymptotic representation of this formula, corresponding to large values of time, is one of the main theoretical expressions used in the hydrodynamic study of wells with a hydraulic fracture. Namely, this expression determines the dependence of the pressure at the bottom of the well on time in the power of $1 / 4$, characteristic of the bilinear filtration mode, and represents a theoretical formula for assessing the fracture conductivity.

It is noted in [2] that due to the complexity of the problem, obtaining corresponding asymptotics for large times from (31) is difficult. Therefore, this asymptotics is determined in the Laplace image space from expression (24) at $x=0$ and small $s$ (large $t$ ) and subsequent transition from the transform image to the original function.

We will demonstrate the way for obtaining asymptotics (31) at large times. In this case $\left(b^{2} t \rightarrow \infty\right)$, the expression (31) can be represented in the form

$$
\bar{P}_{f}(\bar{x}=0, \bar{t}) \approx-\frac{1}{\sqrt{\pi a}} \int_{0}^{\infty} \operatorname{erfc}\left(\frac{u}{2 b \sqrt{\bar{t}}}\right) \frac{d u}{\sqrt{u}} .
$$

Using the substitution $u / 2 b \sqrt{\bar{t}}=z$, we obtain

$$
\bar{P}_{f}(\bar{x}=0, \bar{t})=-\sqrt{\frac{2}{\pi}} \sqrt{\frac{b}{a}}(\bar{t})^{\frac{1}{4}} \int_{0}^{\infty} \operatorname{erfc}(z) \frac{d z}{\sqrt{z}}=\sqrt{\frac{2}{\pi}} \sqrt{\frac{b}{a}}(\bar{t})^{\frac{1}{4}} \frac{2 \Gamma(0.75)}{\sqrt{\pi}}=1.103 \sqrt{\frac{b}{a}}(\bar{t})^{\frac{1}{4}}
$$

where $\Gamma$ is the gamma-function

$$
\Gamma(\xi)=\int_{0}^{\infty} \exp (-u) u^{\xi-1} d u
$$

This expression can be used for determining the fracture conductivity by means of the typical-curve method, i.e., by comparing it with the pressure change curve determined experimentally at the bottom of the well.

The representation of formula (33) in the dimensional form

$$
P_{f}(x=0, t)=P_{0}-1.103 \frac{Q \mu^{3 / 4} t^{1 / 4}}{h_{r} \sqrt{w_{f} k_{f}} \beta_{*}^{1 / 4} k_{r}^{1 / 4}} .
$$

allows to explicitly find the dependence of pressure on the main parameters characterizing the formation, fracture, and fluid (in this formula $\beta_{*}$ is the reservoir elastic capacity coefficient). Analysis shows that the presence of the fracture qualitatively changes the nature of the flow. For example, a classical analog of the expression (34) (with no fracture) is the formula for the pressure on the gallery [8].

$$
P(x=0, t)=P_{0}-1.128 \frac{Q \sqrt{\mu} \sqrt{t}}{b h_{r} \sqrt{k_{r}} \sqrt{\beta_{*}}} .
$$


The amount of fluid flowing from the formation into the fracture is determined from the expression

$$
q=\left.\int_{0}^{\infty} \frac{\partial \bar{P}}{\partial \bar{y}}\right|_{\bar{y}=0} d \bar{x}
$$

Then, taking into account expressions $(12,13)$ and $(18)$,it is possible to find the share in the well flow rate of the volume of fluid entering the fracture from the reservoir

$$
\frac{q}{Q}=1-\exp \left(b^{2} \bar{t}\right) \operatorname{erfc}(b \sqrt{\bar{t}}) .
$$

From this expression, it follows that at the initial stage, the portion of inflow from the fracture prevails in the well production rate, and the portion of inflow from the reservoir increases with time. For example, at $\bar{t}<0.59 / b^{2}$, more than half of the well production rate is determined by the fracture capacity, and in the dimensional form, this condition is as follows:

$$
t<0.59\left(\frac{k_{f}}{k_{r}}\right)^{2} \frac{\kappa_{r} w_{f}^{2}}{\kappa_{f}^{2}} .
$$

Estimates based on this expression show that the manifestation of fracture capacity in the well production rate is noticeable at short times and small values of the fluid mobility $k^{r} / \mu$ in the reservoir.

In the domain $0 \leq z \leq 1$, the erfc function varies from 1 to 0 , therefore, according to the mean value theorem [9], expression (29) can be represented as

$$
\bar{P}_{f}(\bar{x}, \bar{t})=\frac{2 b}{\sqrt{\pi}} \frac{\sqrt{\bar{t}}}{\sqrt{a}} c \int_{0}^{1} \exp \left(-\frac{\bar{x}^{2}}{4 a \overline{t z}^{2}}\right) d z, \quad 0 \leq c \leq 1 .
$$

Obviously, the value of this expression at $c=1$ is a majorant for the solution (29) constructed above in the entire range of variation of $\bar{x}$ and $\bar{t}$. Calculating the integral in (36) and assuming $c=1$, we obtain the well-known formula describing the pressure distribution for plane-parallel fluid filtration in a semi-infinite reservoir, when the flow rate is set at $x=0[8,10,11]$. In dimensional variables, this formula, which also follows from the solution (29) obtained above at $k_{r}=0$ (the absence of fluid crossflow from the reservoir to the fracture), takes the form

$$
P_{f}(x, t)=P_{0}+\frac{\mu Q}{k_{f} w_{f} h_{r}}\left[\operatorname{xerfc}\left(\frac{x}{2 \sqrt{\kappa_{f} t}}\right)-\frac{2 \sqrt{\kappa_{f} t}}{\sqrt{\pi}} \exp \left(-\frac{x^{2}}{4 \kappa_{f} t}\right)\right] .
$$

\section{Summary}

New analytical solutions of the theory of time-dependent fluid flow through a porous reservoir with a vertical hydraulic fracture are given. The solutions are obtained on the basis of the implementation of a model which takes into account fluid flow through a porous reservoir and the fracture compressibility. The model proposed is more generic as compared with the models available in literature. These solutions and their asymptotic 
representations are significant for the theoretical substantiation of hydrodynamic testing methods and for assessing the flow rate of in wells with a hydraulic fracture. The use of the solutions in the case of injection wells makes it possible to describe the dynamics of water flooding of formations with hydraulic fractures, in particular, to determine the speed of fluid movement in the fracture and in the formation when modeling tracer studies.

\section{References}

1. R.D. Kanevskaya, Mathematical Simulation of Oil and Gas Field Development Using Reservoir Hydraulic Fracture. (Moscow: Nedra-Biznestsentr,1999).

2. H.Cinco-Ley, V.F. Samaniego, Transient pressure analysis for fractured wells.J. Petrol. Techonol. 33(9): 1749-1766(1981).

3. D.W. Wong, A.G.Harrington, H. Cinco-Ley, Application of the pressure-derivative function in the pressure-transient testing of fracture wells.Paper SPE 13056, SPE formation Evaluation: 470-480 (1986).

4. G.F.Asalkhuzina, A.Ya.Davletbaev, I.L. Khabibullin, Simulation of differentiation of the reservoir pressure between the injection and producing wells in fields with lowpermeability collectors, Vestn. Bashkir. Univ.21(3): 537-542 (2016).

5. I.L. Khabibullin, A.A. Khisamov, Simulation of unsteady flow through the porous medium in the neighborhood of a well with a vertical hydraulic fracture. Vestn. Bashkir. Univ.22(2): 309-314.(2017).

6. I.L. Khabibullin, A.A. Khisamov, Theory of the bilinear regime of flow through a porous medium in reservoirs with hydraulic fractures. Vestn. Bashkir. Univ.23(4): 958963.2018.

7. A.V. Ditkin, A.P. Prudnikov, Operational Calculus, (Moscow: VysshayaShkola,1975).

8. K.S. Basniev, et al. Underground Hydrodynamics(Moscow-Izhevsk: Institute of Computer Investigations,2006).

9. G.M. Fikhtengol'ts, A Course of Differential and Integral Calculus (Moscow: Nauka,2003).

10. Z.M. Nagaeva, V.Sh. Shagapov, Elastic regime of flow through the porous medium in a fracture located in the oil or gas reservoir. Prikl. Mat. Mekh.81(3): 319-329(2017).

11. I.L.Khabibullin, N.A.Evgrafov, A.A. Khisamov, Simulation of unsteady fluid inflow from a reservoir to a well through hydraulic fracture. Proceedings of the First Summer School-Conference "Physicochemical Hydrodynamics: Models and Applications": 184-192 (2016).

12. Von Doetsch, G.Anleitungzum Practischen Gebrauch der Laplace-Transformation und der Z-Transformation, Munchen, Wien: R. Oldenbourg. 1967. (Moscow: Nauka,1971) 